\title{
AN OPPORTUNISTIC SEARCH FOR EXTRATERRESTRIAL INTELLIGENCE (SETI) WITH THE MURCHISON WIDEFIELD ARRAY
}

\author{
S. J. TingaY ${ }^{1,2}$, C. Tremblay ${ }^{1}$, A. Walsh ${ }^{1}$, and R. Urquhart ${ }^{1}$ \\ ${ }^{1}$ International Centre for Radio Astronomy Research (ICRAR), Curtin University, Bentley, WA 6102, Australia \\ 2 Istituto di Radioastronomia, Istituto Nazionale di Astrofisica, I-40129 Bologna, Italy \\ Received 2016 July 14; revised 2016 July 28; accepted 2016 July 29; published 2016 August 12
}

\begin{abstract}
A spectral line image cube generated from 115 minutes of MWA data that covers a field of view of $400 \mathrm{sq}$, deg. around the Galactic Center is used to perform the first Search for ExtraTerrestrial Intelligence (SETI) with the Murchison Widefield Array (MWA). Our work constitutes the first modern SETI experiment at low radio frequencies, here between 103 and $133 \mathrm{MHz}$, paving the way for large-scale searches with the MWA and, in the future, the low-frequency Square Kilometre Array. Limits of a few hundred mJy beam ${ }^{-1}$ for narrowband emission $(10 \mathrm{kHz})$ are derived from our data, across our $400 \mathrm{sq}$. deg. field of view. Within this field, 45 exoplanets in 38 planetary systems are known. We extract spectra at the locations of these systems from our image cube to place limits on the presence of narrow line emission from these systems. We then derive minimum isotropic transmitter powers for these exoplanets; a small handful of the closest objects $\left(10 \mathrm{~s} \mathrm{of} \mathrm{pc)} \mathrm{yield} \mathrm{our} \mathrm{best} \mathrm{limits} \mathrm{of} \mathrm{order} 10^{14} \mathrm{~W}\right.$ (Equivalent Isotropic Radiated Power). These limits lie above the highest power directional transmitters near these frequencies currently operational on Earth. A SETI experiment with the MWA covering the full accessible sky and its full frequency range would require approximately one month of observing time. The MWA frequency range, its southern hemisphere location on an extraordinarily radio quiet site, its very large field of view, and its high sensitivity make it a unique facility for SETI.
\end{abstract}

Key words: instrumentation: interferometers - planets and satellites: detection - radio lines: planetary systems techniques: spectroscopic

\section{INTRODUCTION}

The first modern Search for ExtraTerrestrial Intelligence (SETI) was undertaken at radio wavelengths in 1960 at Green Bank, West Virginia (Drake 2008), targeting two stars, Tau Ceti and Epsilon Eridani at frequencies near the $21 \mathrm{~cm}$ line of neutral hydrogen.

In the decades since, SETI programs have continued to be undertaken at radio wavelengths, on the basis that highly sensitive radio telescopes exist for astronomy, the radio band is the cornerstone of communications technologies on Earth, and it could be reasonably assumed that a similar technology path has been taken by extraterrestrial civilizations. In 1985, the Million-channel ExtraTerrestrial Assay (META) was established at Harvard University (Leigh \& Horowitz 1997), also near $1.4 \mathrm{GHz}$. META was upgraded to the Billion-channel ExtraTerrestrial Assay in 1995, in the extended frequency range of 1.4-1.7 GHz. The Search for Extraterrestrial Radio Emissions from the Nearby Developed Intelligent Populations (SERENDIP) program was established in 1978 and has evolved considerably since then (Werthimer et al. 2001). The SERENDIP program also gave rise to projects such as SETI@ home (Korpela et al. 2009) and Southern SERENDIP (Stootman et al. 2000), a survey conducted using the Parkes radio telescope. A novel targeted search using Very Long Baseline Interferometry observations of the Gliese 581 star system is described by Rampadarath et al. (2012). The Allen Telescope Array has been used extensively for a range of SETI experiments over the last decade (Welch et al. 2009). Recently the ATA has conducted SETI experiments targetting over 9200 exoplanets (Harp et al. 2016a, 2016b). See Garrett (2015) for a recent review of aspects of SETI experiments at radio wavelengths. For general reviews, see Tarter (2003) and Siemion et al. (2015).
The SETI experiments conducted at radio wavelengths to date have generally focused on the $1.4-1.7 \mathrm{GHz}$ range, the socalled "water hole" between the prominent radio spectral lines due to neutral hydrogen $(\mathrm{H})$ and hydroxyl $(\mathrm{OH})$. However, many other radio frequencies are also viable for SETI experiments. One radio frequency range that has opened up in recent years is in the tens to hundreds of MHz. Powerful multi-purpose, next-generation low-frequency radio telescopes such as the Low-frequency Array (LOFAR: van Haarlem et al. 2013) and the Murchison Widefield Array (MWA: Lonsdale et al. 2009; Tingay et al. 2013) are precursors and pathfinders for the billion dollar Square Kilometre Array (SKA: Dewdney et al. 2009) over the next decade. A key science program for the SKA is the "cradle of life" (Hoare et al. 2015), including comprehensive and ambitious SETI experiments (Siemion et al. 2015). Loeb \& Zaldarriaga (2007) discuss the prospects for SETI experiments using facilities such as the MWA, LOFAR, and the low-frequency SKA, instruments that are primarily designed to search for the redshifted neutral hydrogen line from the Epoch of Reionization. Rahvar (2015) discusses the potential for utilising microlensing for SETI programs at radio wavelengths, with particular reference to the MWA and SKA. LOFAR, operating in the ranges of $30-80 \mathrm{MHz}$ and $120-240 \mathrm{MHz}$, launched a SETI project in $2010,{ }^{3}$ although no results have thus far been published.

The MWA operates in the frequency range of $80-300 \mathrm{MHz}$ on an exceptionally radio quiet site in Western Australia. The high sensitivity of the MWA, its radio quiet location (Offringa et al. 2015), its frequency range, its access to the Southern Hemisphere, and its exceptionally large field of view (hundreds

\footnotetext{
3 https://www.astron.nl/about-astron/press-public/news/lofar-opens-lowfrequency-universe-and-starts-new-seti-search/lofar-o
} 
of square degrees) make it a unique facility for exploratory SETI experiments. As Garrett (2015) points out, the emergence of new radio telescopes with very large fields of view opens up new areas of parameter space for SETI experiments. For example, within a single $>500 \mathrm{sq}$. deg. field of view typical for the MWA, on average tens of stellar systems within 50 lightyears will be accessible (ESA 1997). Based on recent results showing that planets are the norm rather than the exception, for example on average $1.0 \pm 0.1$ planets per $\mathrm{M}$ dwarf star in our Galaxy (Swift et al. 2013), one would therefore expect dozens of nearby (within 50 lightyears) planets in a single MWA field of view and far greater numbers of more distant planets. The MWA field of view, therefore, results in a significant multiplex advantage that can be exploited for SETI experiments.

While facilities such as the MWA, LOFAR, and the SKA will open up unprecedented parameter space for new SETI experiments, it is worth noting that the very first consideration of low radio frequency SETI came approximately 60 years before the first modern experiments described in Drake (2008), at the dawn of radio communications. In the late 1800s and early 1900s, Guglielmo Marconi ${ }^{4}$ and Nicola Tesla ${ }^{5}$ believed that radio waves could be used to communicate with civilizations on Mars (the widespread belief in the existence of Martian canals persisted at the time) and both claimed to have detected potential signals from that planet. In 1924, an experiment to listen for signals was organized by the US Navy during the opposition of Mars that year, coordinated with a planned cessation of terrestrial radio broadcasts. ${ }^{6}$ These very first low radio frequency experiments returned null results.

In this Letter, we present a first, and opportunistic, SETI pilot experiment with the MWA, in the frequency range $103-133 \mathrm{MHz}$, placing limits on narrowband radio emission toward 38 known planetary systems. The experiment is opportunistic in the sense that the observations were undertaken for a spectral line survey of the Galactic Plane that is ongoing; utility of the data for a SETI experiment was realized post-observation. We use this pilot study to motivate a deeper and significantly larger SETI experiment with the MWA, that could use the full $80-300 \mathrm{MHz}$ frequency range and survey the entire southern sky (majority of the Milky Way) visible from Western Australia.

The field of SETI has recently received a substantial boost, with the "Breakthrough Listen" project recently initiated." Novel and diverse SETI experiments that sit on the path to utilization of the SKA for SETI, such as described here, are likely to be useful contributors to such initiatives.

\section{OBSERVATIONS AND DATA ANALYSIS}

The MWA system is described in Tingay et al. (2013), so we provide only a brief summary of the salient aspects of the observations. Observations with the MWA took place on 2014 July 25. Dual-polarization data were obtained in a $30.72 \mathrm{MHz}$ contiguous bandwidth (consisting of $24 \times 1.28 \mathrm{MHz}$ contiguous coarse channels), centered at $119.7 \mathrm{MHz}$, with $10 \mathrm{kHz}$ frequency resolution (thus 128 fine channels per $1.28 \mathrm{MHz}$

\footnotetext{
4 Reported extensively in the media at the time, for example on page 3 of the New York Tribune, 1921 September 2: http:chroniclingamerica.loc.govlccn/ sn830302141921-09-02ed-1seq-3.

5 http:www.teslasociety.commars.pdf.

6 http:www.lettersofnote.com200911 prepare-for-contact.html

7 http://www.breakthroughinitiatives.org/
}

coarse channel). Observations took place in 5 minute segments over a total of 115 minutes, with pointing coordinates of R.A. $=17^{\mathrm{h}} 45^{\mathrm{m}} 40^{\mathrm{s}} .036$ and decl. $=-29^{\circ} 00^{\prime} 28^{\prime \prime} .17$ and a field of view (primary beam) full width at half maximum (FWHM) of $\sim 30^{\circ}$ at a resolution (synthesized beam) of $3^{\prime}$. However, only 400 sq. deg. were imaged and searched, in order to make this first search a manageable size.

The edges of each coarse channel suffer from aliasing, requiring a number of fine channels on each coarse channel edge to be flagged. This resulted in approximately $78 \%$ of the 3072 fine channels being imaged. The central fine channel of each coarse channel was flagged, as they contain the DC component of the filterbank. Automated flagging of radio frequency interference (RFI) was performed using AO Flagger (Offringa et al. 2012) and a small number of fine channels were manually removed. In total, less than $0.5 \%$ of data were removed due to RFI, consistent with previous examinations of the RFI environment of the MWA (Offringa et al. 2015). As described in Tingay et al. (2015), AO Flagger only removes the strongest RFI, well above any limits on SETI signals discussed below. We also note that the RFI removed all corresponds to known terrestrial transmitter frequencies (generally FM radio and digital TV broadcast frequencies).

Calibration and imaging proceeded using the techniques described in Hurley-Walker et al. (2014). This includes using WS Clean (Offringa et al. 2014), using w-stacking to take into account the w-terms, to clean and image each individual fine channel with Briggs weighting set to -1 , a compromise between natural and uniform weighting to balance signal to noise and image resolution. The 100 individual fine channels from each coarse channel are imaged using 5.7 pixels per synthesised beam FWHM and were combined to produce a single data cube which was then converted into a MIRIAD (Sault et al. 1995) file format. In MIRIAD, all of the five-minute observations were timeaveraged together to form a single data cube, representing an effective 82 minutes of integration time.

A continuum-subtracted image cube was produced, removing $99 \%$ of continuum emission using custom software. The field of view imaged for the cube was 400 sq. deg, covering the R.A. range $17^{\mathrm{h}} 03^{\mathrm{m}} 24^{\mathrm{s}}-18^{\mathrm{h}} 28^{\mathrm{m}} 06^{\mathrm{s}}$ and the decl. range $-18^{\circ}$ $34^{\prime} 48^{\prime \prime}$. to $-38^{\circ} 30^{\prime} 44^{\prime \prime}$. The rms noise levels in the continuumsubtracted cube (Table 1) were in line with theoretical noise estimates, calculated from the system description in Tingay et al. (2013).

Although an analytic model for a dipole over a ground screen has been used previously for primary beam correction with MWA data Hurley-Walker et al. (2014), we made no correction for the beam model in the creation of these data cubes, since this experiment is primary a detection experiment. To estimate the variation in expected flux densities, the data from the Molonglo Reference Catalog (MRC; Large et al. 1981), NRAO VLA Sky Survey (Condon et al. 1998), and Parkes-MIT-NRAO survey (Griffith et al. 1994) were compared to the flux density recovered for extragalactic sources in a non-continuum-subtracted image. This showed an error in the flux densities between $7 \%$ and $38 \%$ depending on angular distance from the phase center. In order to be conservative, the upper limits listed in Table 1 include a $40 \%$ error in the flux density scale.

A search of the field for exoplanets, based on the Kepler catalog (Akeson et al. 2013), returned 38 known planetary systems containing 45 exoplanets. The systems are listed in 
Table 1

The 38 Known Exoplanet Systems in the MWA Field of View

\begin{tabular}{|c|c|c|c|c|c|}
\hline \# System & R.A. & Decl. & rms (mJy) & Dist. (pc) & $\mathrm{P}\left(10^{13} \mathrm{~W}\right)$ \\
\hline GJ $667 \mathrm{C}$ & 259.7451 & -34.9968 & 529 & 6.8 & $<4$ \\
\hline HD 156846 & 260.1429 & -19.3337 & 461 & 49 & $<185$ \\
\hline HD 164604 & 270.7789 & -28.5606 & 488 & 38 & $<117$ \\
\hline HD 169830 & 276.9562 & -29.8169 & 453 & 36 & $<98$ \\
\hline MOA 2007-BLG-192L & 272.0158 & -27.1501 & 457 & 1000 & $<8 \times 10^{4}$ \\
\hline MOA 2007-BLG-400L & 272.4249 & -29.2242 & 470 & 5800 & $<3 \times 10^{6}$ \\
\hline MOA 2008-BLG-310L & 268.5605 & -34.7781 & 461 & 6000 & $<3 \times 10^{6}$ \\
\hline MOA 2008-BLG-379L & 269.7060 & -30.1969 & 443 & 3300 & $<8 \times 10^{5}$ \\
\hline MOA 2009-BLG-266L & 267.0081 & -35.0054 & 454 & 3040 & $<7 \times 10^{5}$ \\
\hline MOA 2009-BLG-319L & 271.7422 & -26.8197 & 465 & 6100 & $<3 \times 10^{6}$ \\
\hline MOA 2009-BLG-387L & 268.4616 & -33.9903 & 448 & 5690 & $<2 \times 10^{6}$ \\
\hline MOA 2010-BLG-073L & 272.5473 & -26.5229 & 489 & 2800 & $<6 \times 10^{5}$ \\
\hline MOA 2010-BLG-328L & 269.4963 & -30.7152 & 467 & 810 & $<5 \times 10^{4}$ \\
\hline MOA 2010-BLG-477L & 271.5310 & -31.4545 & 460 & 2300 & $<4 \times 10^{5}$ \\
\hline MOA 2011-BLG-262L & 270.0978 & -31.2453 & 474 & 7000 & $<4 \times 10^{6}$ \\
\hline MOA 2011-BLG-293L & 268.9140 & -28.4768 & 480 & 7720 & $<5 \times 10^{6}$ \\
\hline MOA 2011-BLG-322L & 271.2233 & -27.2209 & 469 & 7560 & $<4 \times 10^{6}$ \\
\hline MOA-bin-1L & 261.7925 & -29.7940 & 452 & 5100 & $<2 \times 10^{6}$ \\
\hline OGLE 2003-BLG-235L & 271.3181 & -28.8950 & 478 & 5200 & $<2 \times 10^{6}$ \\
\hline OGLE 2005-BLG-169L & 271.5222 & -30.7326 & 469 & 2700 & $<6 \times 10^{5}$ \\
\hline OGLE 2005-BLG-390L & 268.5800 & -30.3773 & 474 & 6600 & $<3 \times 10^{6}$ \\
\hline OGLE 2005-BLG-71L & 267.5407 & -34.6732 & 456 & 3200 & $<8 \times 10^{5}$ \\
\hline OGLE 2006-BLG-109L & 268.1438 & -30.0878 & 469 & 1510 & $<2 \times 10^{5}$ \\
\hline OGLE 2007-BLG-368L & 269.1082 & -32.2374 & 464 & 5900 & $<3 \times 10^{6}$ \\
\hline OGLE 2008-BLG-355L & 269.7867 & -30.7595 & 476 & 6800 & $<4 \times 10^{6}$ \\
\hline OGLE 2008-BLG-92L & 266.8726 & -34.7266 & 455 & 8100 & $<5 \times 10^{6}$ \\
\hline OGLE 2011-BLG-251L & 264.5591 & -27.1361 & 469 & 2570 & $<5 \times 10^{5}$ \\
\hline OGLE 2011-BLG-265L & 269.4488 & -27.3945 & 506 & 4380 & $<2 \times 10^{6}$ \\
\hline OGLE 2012-BLG-26L & 263.5779 & -27.1428 & 453 & 4080 & $<1 \times 10^{6}$ \\
\hline OGLE 2012-BLG-358L & 265.6949 & -24.2610 & 443 & 1760 & $<2 \times 10^{5}$ \\
\hline OGLE 2012-BLG-406L & 268.3257 & -30.4712 & 472 & 4970 & $<2 \times 10^{6}$ \\
\hline OGLE 2013-BLG-102L & 268.0295 & -31.6906 & 457 & 3040 & $<7 \times 10^{5}$ \\
\hline OGLE 2013-BLG-341L B & 268.0312 & -29.8461 & 475 & 1161 & $<1 \times 10^{5}$ \\
\hline OGLE 2014-BLG-124L & 270.6217 & -28.3963 & 470 & 4100 & $<1 \times 10^{6}$ \\
\hline OGLE-TR-056 & 269.1480 & -29.5392 & 478 & 1500 & $<2 \times 10^{5}$ \\
\hline OGLE-TR-10 & 267.8677 & -29.8764 & 475 & 1500 & $<2 \times 10^{5}$ \\
\hline SWEEPS-11 & 269.7583 & -29.1982 & 470 & 8500 & $<6 \times 10^{6}$ \\
\hline SWEEPS-4 & 269.7247 & -29.1891 & 473 & 8500 & $<6 \times 10^{6}$ \\
\hline
\end{tabular}

Note. Column 1: exoplanet system; column 2: R.A. (deg); column 3: decl. (deg); column 4: rms (mJy beam ${ }^{-1}$ ); column 5: distance (pc); and column 6: upper limit on isotropic transmitter power in units of $10^{13} \mathrm{~W}$.

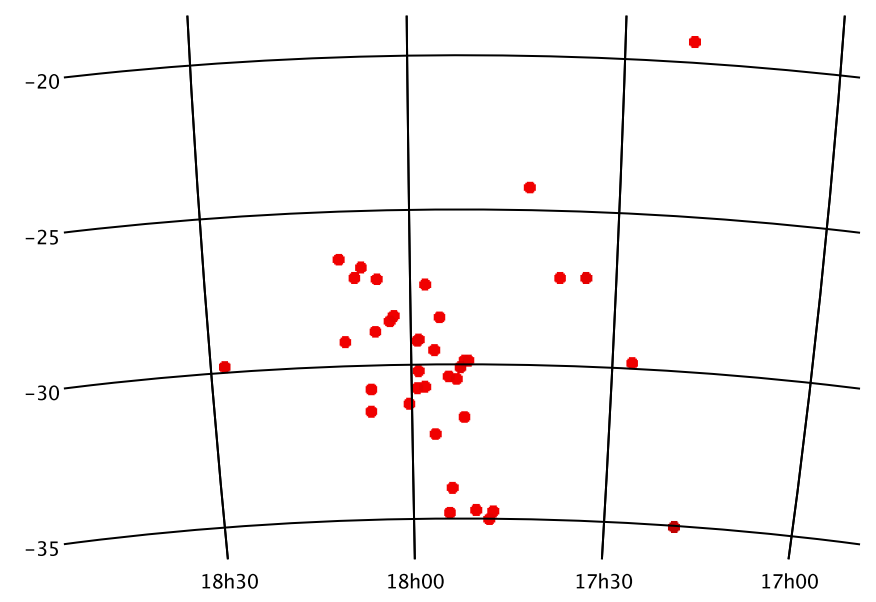

Figure 1. Distribution of the 38 known exoplanet systems in the MWA field of view.
Table 1 and are shown in relation to the MWA field of view in Figure 1.

The MWA data cube was searched at the locations of each of these exoplanet systems and spectra were extracted from these locations. No significant narrowband signals were detected above a $5 \sigma$ level in any of these spectra. Table 1 lists the rms from the spectra at each of the exoplanet system locations and the corresponding $1 \sigma$ limits on the inferred isotropic transmitter power required at the distance of the exoplanet system (ranging between $10^{14}$ and $10^{20} \mathrm{~W}$ ). Figure 2 shows example spectra extracted from the MWA data cube, representing the four closest stars in Table 1: GJ 667; HD 156846; HD 164604; and HD 169830.

GJ 667, at $6.8 \mathrm{pc}$, is the closest star in Table 1 . The closest recorded star to Earth in our field is 36 Ophiuchi, at a distance of $5.9 \mathrm{pc}$, which is not significantly closer than GJ 667 when considering the calculation of limits on transmitter power in 

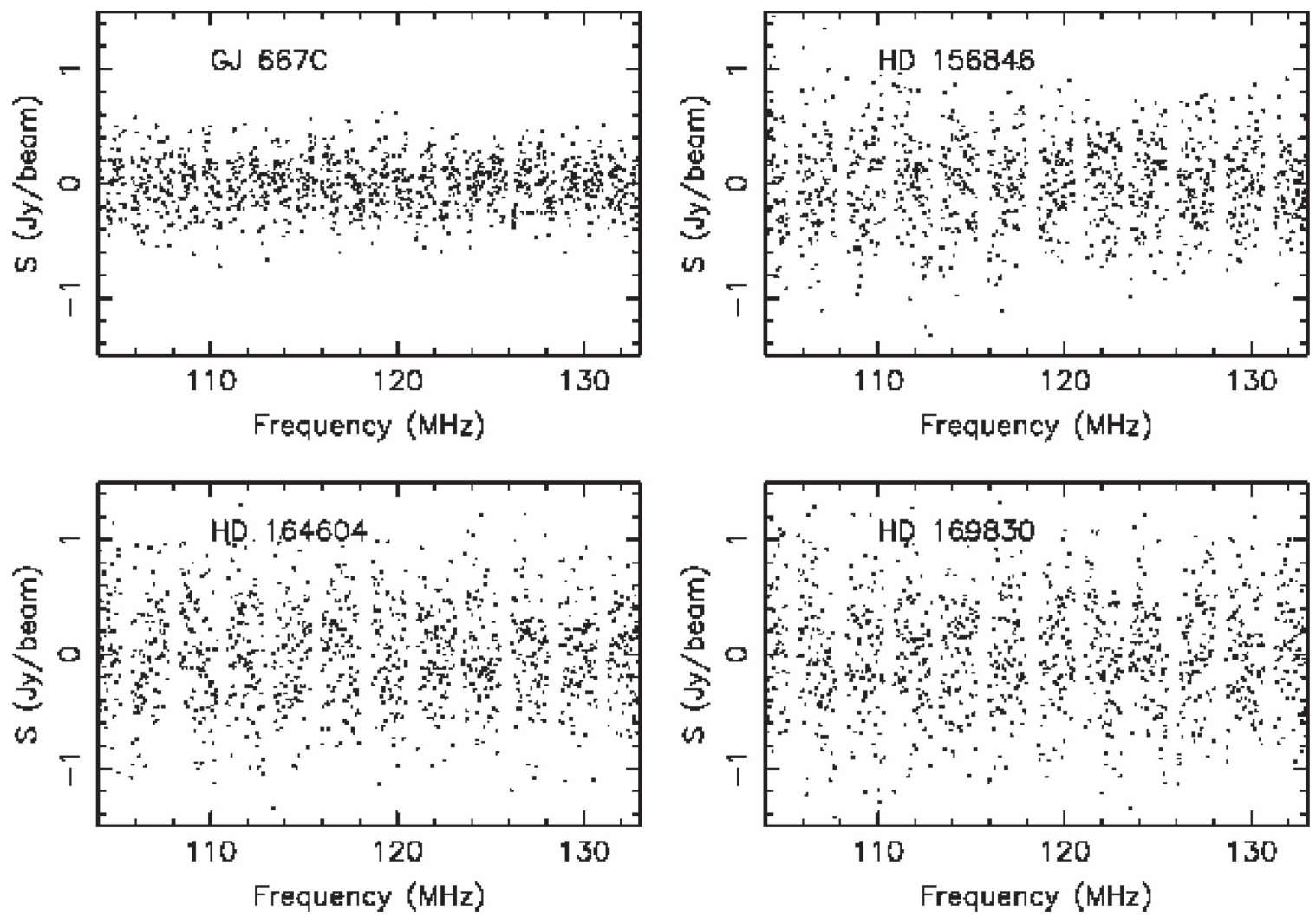

Figure 2. MWA spectra for the four closest exoplanet systems listed in Table 1. Areas of flagged fine channels on coarse channel edges are evident as gaps in the spectra.

Table 1. For the typical spectral rms in Table 1, four to five hundred $\mathrm{mJy}_{\text {beam }}{ }^{-1}$, the corresponding limit for the nearest extrasolar star to the Earth (proxima Centauri, $1.3 \mathrm{pc}-$ not in our field) would be approximately $10^{13} \mathrm{~W}$.

\section{DISCUSSION AND CONCLUSION}

The great majority of exoplanet systems listed in Table 1 are at large distances, yielded from microlensing experiments toward the Galactic Center, meaning that the observational limits on detectable transmitter power from the MWA are very high, inferred isotropic powers of $10^{17}-10^{19} \mathrm{~W}$. Even if a directional transmitting antenna is assumed, with a gain similar to low-frequency over-the-horizon (OTH) radar transmitters on Earth, the limits on transmitter power are only reduced by factors of order 100 .

A small handful of exoplanet systems in Table 1 are close enough that the inferred isotropic transmitter powers are of order $10^{13}-10^{14} \mathrm{~W}$. These are still very large in terms of transmitters on Earth. The highest power low-frequency transmitters on Earth are the OTH-backscatter radars used for military surveillance; these typically operate in the $5-30 \mathrm{MHz}$ range and have transmitter powers of order $1 \mathrm{MW}$. For example, the Jindalee Operation Radar Network in Australia has a transmitter power of $560 \mathrm{~kW}$ (Colegrove 2000) and similar installations in the US, such as the in the AN/FPS-118 OTH-Backscatter radar, ${ }^{8}$ have transmitter powers of $1 \mathrm{MW}$ (but can range up to $10 \mathrm{MW}$ ). In the latter case of the US system, the Effective Radiated Power is $100 \mathrm{MW}$, still a factor of $\sim 10^{5}$ below the limits for the nearest exoplanet systems in Table 1.

\footnotetext{
http://www.globalsecurity.org/wmd/systems/an-fps-118.htm
}

Even the addition of the signals from the ensemble of global array of OTH radars fall well below our limits. Loeb \& Zaldarriaga (2007) summarize other Earth-based transmitter characteristics relevant to low-frequency telescopes.

The most powerful transmission ever deliberately broadcast into space was the Arecibo message, which was directed as a purposeful communication at the globular cluster M13, in a $10 \mathrm{~Hz}$ bandwidth at $2380 \mathrm{MHz}$ (NAIC 1975). This transmission had an equivalent isotropic transmission of $20 \times 10^{9} \mathrm{~W}$. Taking the narrow bandwidth into account (and ignoring the large difference in frequency), this transmission once again falls below the limits calculated in Table 1 .

In the MWA frequency range, McKinley et al. (2013) previously estimated the Equivalent Isotropic Power of FM radio transmissions from the Earth to be $77 \mathrm{MW}$. This estimate was made by measuring the amount of stray FM radio signal reflected off the Earth's moon. Again, this isotropic power is well below the limits in Table 1 .

Of course, these projections of Earth-based technologies discount the possibility that higher power and/or more highly directive antenna technologies are utilized by advanced extraterrestrial civilizations for communications or remote sensing applications.

While the inferred transmitter powers in Table 1 are high compared wtih the most powerful low-frequency transmitters on Earth, this study has nonetheless provided the most comprehensive search for narrowband transmissions from exoplanets in this frequency range. Due to the southern, RFI free location of the MWA, its operational frequency range, and its wide field of view, the MWA provides a unique capability for future SETI projects. 
This experiment examined one field of view of 400 sq. deg. in a $30.72 \mathrm{MHz}$ frequency band. To perform a SETI experiment to the same depth as achieved here, but over the full MWA frequency range $(80-300 \mathrm{MHz})$, and over the full accessible sky from Western Australia, would require approximately one month of observing time. This is an entirely feasible goal for the near future (three times deeper would require of order a year of observing). Moreover, to relieve the restriction of $10 \mathrm{kHz}$ frequency resolution present in the current experiment, it is possible to record voltage data from the MWA and reconstruct coherent beams at far higher spectral resolution to target individual exoplanet systems Tremblay et al. (2015b). For example, generating $1 \mathrm{~Hz}$ channels from coherent beams across the full array would yield a factor of 100 improvement in sensitivity (assuming a $1 \mathrm{~Hz}$ transmission bandwidth), compared to the current experiment. Such a mode could be run communally with the large-scale survey described above, for a selected list of target systems.

The current experiment and the capabilities of the MWA provide a clear path to the far greater capabilities of the lowfrequency component of the SKA, which will be built at the same location as the MWA and have a spectral sensitivity some tens of times greater than the MWA. The radio quiet nature of the MWA/SKA site in enabling SETI experiments at low frequencies (especially through the FM band), as demonstrated here, bodes well for SETI experiments with the SKA.

This scientific work makes use of the Murchison Radioastronomy Observatory, operated by CSIRO. We acknowledge the Wajarri Yamatji people as the traditional owners of the Observatory site. We thank Ian Morrison and Andrew Siemion for discussions regarding a draft of this Letter. Support for the operation of the MWA is provided by the Australian Government Department of Industry and Science and Department of Education (National Collaborative Research Infrastructure Strategy: NCRIS), under a contract to Curtin University administered by Astronomy Australia Limited. We acknowledge the iVEC Petabyte Data Store and the Initiative in Innovative Computing and the CUDA Center for Excellence sponsored by NVIDIA at Harvard University.

Facility: MWA.

\section{REFERENCES}

Akeson, R. L., Chen, X., Ciardi, D., et al. 2013, PASP, 125, 989
Colegrove, S. B. 2000, in The Record of the IEEE 2000 International Radar Conference (Piscataway, NJ: IEEE), 825

Condon, J. J., Cotton, W. D., Greisen, E. W., et al. 1998, AJ, 115, 1693

Dewdney, P. E., Hall, P. J., Schilizzi, R. T., \& Lazio, T. J. L. W. 2009, IEEEP, 97, 1482

Drake, F. 2008, in ASP Conf. Ser. 395, Frontiers of Astrophysics: A Celebration of NRAOs 50th Anniversary, ed. A. H. Bridle, J. J. Condon, \& G. C. Hunt, (San Francisco, CA: ASP), 213

ESA, 1997, The Hipparcos and Tycho Catalogues (ESA SP-1200; Noordwijk: ESA)

Garrett, M. A. 2015, AcAau, 113, 8

Griffith, M. R., Wright, A. E., Burke, B. F., \& Ekers, R. D. 1994, ApJS, 90, 179

Harp, G. R., Richards, J., Shostak, S., et al. 2016a, ApJ, 825, 155

Harp, G. R., Richards, J., Tarter, J. C., et al. 2016b, ApJ, submitted (arXiv:1607.04207)

Hoare, M., Perez, L., Bourke, T. L., et al. 2015, in Proc. Advancing Astrophysics with the Square Kilometre Array (AASKA14) (Giardini Naxos, Italy), 115, http://pos.sissa.it/cgi-bin/reader/conf.cgi?confid $=215$

Hurley-Walker, N., Morgan, J., Wayth, R. B., et al. 2014, PASA, 31, e045

Korpela, E. J., Anderson, D. P., Bankay, R., et al. 2009, in ASP Conf. Ser. 420, Bioastronomy 2007: Molecules, Microbes and Extraterrestrial Life, ed. K. J. Meech et al. (San Francisco, CA: ASP), 431

Large, M. I., Mills, B. Y., Little, A. G., Crawford, D. F., \& Sutton, J. M. 1981, MNRAS, 194, 693

Leigh, D., \& Horowitz, P. 1997, in IAU Coll. 161, Astronomical and Biochemical Origins and the Search for Life in the Universe, ed. C. Batalli Cosmovici, S. Bowyer, \& D. Werthimer (Bologna, Italy), 601

Loeb, A., \& Zaldarriaga, M. 2007, JCAP, 01, 020

Lonsdale, C. J., Cappallo, R. J., Morales, M. F., et al. 2009, IEEEP, 97, 1497

McKinley, B., Briggs, F., Kaplan, D. L., et al. 2013, AJ, 145, 23

Offringa, R. A., de Bruyn, A. G., Zaroubi, S., et al. 2012, MNRAS, 422, 563

Offringa, R. A., McKinley, B., Hurley-Walker, N., et al. 2014, MNRAS, 444,606

Offringa, R. A., Wayth, R. B., Hurley-Walker, N., et al. 2015, PASA, 32, e008

Rahvar, S. 2015, ApJ, in press (arXiv:1509.05504)

Rampadarath, H., Morgan, J. S., Tingay, S. J., \& Trott, C. M. 2012, AJ, 144, 38

Sault, R. J., Teuben, P. J., \& Wright, M. C. H. 1995, in ASP Conf. Ser. 77, Astronomical Data Analysis Software and Systems IV, ed. R. A. Shaw, H. E. Payne, \& J. J. E. Hayes (San Francisco, CA: ASP), 433

Siemion, A. P. V., Benford, J., Cheng-Jin, J., et al. 2015, PoS(AASKA14)116, in press (arXiv:1412.4867)

Staff at the National Astronomy and Ionosphere Centre, 1975, Icar, 26, 462

Stootman, F. H., De Horta, A. Y., Oliver, C. A., \& Wellington, K. J. 2000, in ASP Conf. Ser. 213, Bioastronomy 99, ed. G. Lemarchand \& K. Meech, (San Francisco, CA: ASP), 491

Swift, J. J., Johnson, J. A., Morton, T. D., et al. 2013, ApJ, 764, 105

Tarter, J. 2003, ARA\&A, 39, 511

Tingay, S. J., Goeke, R., Bowman, J. D., et al. 2013, PASA, 30, e007

Tingay, S. J., Trott, C. M., Wayth, R. B., et al. 2015, AJ, 150, 199

Tremblay, S., Ord, S. M., Bhat, N. D. R., et al. 2015b, PASA, 32, e005

van Haarlem, M. P., Wise, M. W., Gunst, A. W., et al. 2013, A\&A, 556, A2

Welch, J., Backer, D., Blitz, L., et al. 2009, IEEEP, 97, 1438

Werthimer, D., Anderson, D., Bowyer, C. S., et al. 2001, Proc. SPIE, 4273, 104 